\title{
Two-way Interaction between Modern Consumer Psychology and Packaging Design
}

\section{Qiu Li}

\author{
Yunnan Engineering Vocation College, Anning, Yunnan, 650304
}

\begin{abstract}
Keywords: consumer psychology; packaging design; two-way interaction
\end{abstract}
\begin{abstract}
The society today is a society with a surplus of materials. Consumption philosophy of people today is also very different from the past. In the past, good prices and low prices were people's choices. Now, the majority of people are spending for their lives. For modern consumption, the main function of packaging design is to beautify and convey information, as well as to highlight the information and value of the product. It is believed that good packaging design not only shall pay attention to the basic principles of design, but also focus on the study of consumer psychological activities in order to stand out in the same category of goods. From the consumer's consumer motivation and consumer psychology, the two-way interaction between modern consumer psychology and packaging design is theoretically discussed.
\end{abstract}

\section{Consumer Motivation}

The most direct goal of product packaging is to inspire consumers to make purchases. The first consideration in formulating a product packaging plan should be this goal. Second, even if consumers are not ready to purchase such products, they should also prompt them to make a good impression on the brand, packaging and brand of the product and the manufacturer. People's actions are generally dictated by certain subjective internal causes, namely motivation, and motivation is closely related to needs. Motivation is the reflection of needs under certain conditions, which is transformed by human needs. In other words, people act in order to meet certain needs. Consumers buy something from the store because they need it. As winter approaches, people will go to the shops to buy things like down coats or overcoats to resist the cold. Motivation comes from the need to transform, but the needs of people do not necessarily translate into motives that motivate people to act. Needs are often experienced in the form of desire. With the motivation to satisfy the desire, people often take purchasing actions. Only at this time can the need be truly translated into motivation and become the driving force for people's buying behavior.

People's needs are hierarchical, and many psychologists have conducted in-depth discussions. Maslow, an American psychologist. He divides people's needs into five levels:

(1) Physiological needs: eating, drinking, air, etc.;

(2) Security needs: the need for freedom in danger and fear, etc.;

(3) The need for belonging and love: to be loved by relatives and friends, and to establish a family;

(4) The need for respect: The need for honorable achievement

(5) Self-realistic needs. Among them, self-reality needs include the need for knowledge; aesthetic needs; the need for their own growth and development; the need to fully develop their talents. Some consumers buy goods, on the one hand, for use, and on the other hand to show their status, education, and appreciation [1]. Therefore, when formulating the positioning of different commodity packaging designs, the needs and motivation types of consumers should be investigated and researched in order to carry out targeted packaging design.

\section{Consumer Psychology}

In consumption, there is a series of psychological activities during the purchase process from the consumer's motivation to the specific purchase behavior. 
(1) Cognitive Process: Consumers always buy goods from the process of understanding the merchandise. It is the process of consumers' contact, understanding and mastering of commodity information and is the basis and precursor of purchasing activities. In an assortment of supermarkets, consumers are the first to see the packaging of goods, so the goods must highlight new, extraordinary, special, showing a unique packaging materials, patterns, colors, logos, so that the company's product packaging. It is particularly eye-catching in shopping malls, which has laid a good foundation for further understanding and consumption of this product. With the deepening of feelings, the analysis and synthesis of various sensory materials will form a reflection of consumers' overall characteristics of the product. This is perception. As the outer garment of a product, the package should show as much as possible the various conditions inherent in the product [2].

(2) Emotional process: The process of consumers' psychological activity is the process of changing emotions. This is because consumers are living organisms and they are in a complex and changing social environment. In the purchasing process, there are many factors that affect the emotional changes of consumers, such as the influence of purchasing environment (such as supermarket environment, goods decoration, the number of shoppers, background music, etc.), the influence of goods (such as the price, variety, quality, etc. of the goods), the influence of individual consumer emotions. This is the process of subjective experiences and feelings of consumers on commodities, and this psychological activity process has formed. The consumer's subjective attitude toward the product. This process is a key stage in the process of purchasing psychological activities.

(3) The Will Process: The process of consumers' psychological activities includes not only cognitive processes, emotional processes, but also the process of will. The actual purchase process of the consumer is determined by the determination of the will. The relevant product information obtained through the packaging of goods is summed up and summarized at this stage, and combined with its own needs, it is decided to take a purchase action.

\section{Psychological Strategies for Packaging Design}

Packaging in the circulation of goods has always been an important promotional tool, and it is also the media that best meets consumers' psychological demands. One of its important missions is to attract consumers' attention and generate the desire to purchase. At this time, they will carry out the cognition process of goods, that is, the process of familiarity and consideration. Pursuit of desire can be said to be the basic motivation for purchasing, in which the appearance of product packaging, internal quality, and price have the greatest impact.

(1) According to consumption habits. Should pay attention to the consumer's consumption habits, according to the characteristics of the product itself, designed to meet the majority of people's spending habits of packaging. For example, many customers are looking for convenience in shopping. If they use transparent or window-packed foods, they can be easily picked up. Combined packaged gift baskets can be easily used; soft-packed drinks can be easily carried and so on.

(2) Designed according to the age of the consumer object. Commodities usually have a certain range of age sales. Therefore, in the design of commodity packaging, rational design should be carried out according to the ages of the consumers. The packaging of goods sold for the elderly should be biased towards simplicity, generosity and simplicity. The products targeted at young people are different in terms of packaging design. They are seeking new ideas, seeking differences, and seeking curiosity. They strive to find opportunities to express themselves and guide the trend. As for products that target children, they should pay attention to their psychology of seeking.

(3) Differences in design according to the cultural level and economic income of the target consumer will usually lead to different consumer appreciation and requirements for the packaging of the product [3]. For consumers with relatively high economic income, in addition to the higher requirements for product quality, they usually pay attention to taste and grade in packaging design, and strive to exquisitely and elegantly packaged to highlight the aesthetic and taste of consumers. For consumers with relatively low economic income, the packaging of goods is usually in a subordinate position. Such consumers pay more attention to the quality of goods, and there is no excessive demand for the packaging of goods. 


\section{Problems in Packaging Design}

(1) The packaging design does not show the sales proposition of the company's products. The products can satisfy the consumers' demands and belongings through the packaging design. When a product's packaging design lacks the theme, it is easy to lose the theme in the fierce market competition. Competitors, this is the product sales claims are inhibited.

(2) The design of the packaging design and the connotation of the product packaging design of the enterprise products in the South and the South is borne by the printing factory. The design capabilities of the printing factory are often uneven, and the design of some small printing companies is usually eye-popping.

(3) The packaging design of products is simplistic. Some enterprises' products are often used to take "shortcuts" in order to achieve differentiated sales. That is, they do not create differences through the products themselves. Instead, they create differences through the "luxury route" of packaging design [3].

(4) The packaging design of the product does not meet the consumer's behavioral habits. A brand of functional beverages is used in the country. The target consumer is a person with a large amount of exercise, and the packaging material is a glass bottle. There is a problem here, glass itself is fragile, in the behavioral habits of their own body protection, glass bottle of functional sports drinks is obviously very difficult to be respected. In addition, some field snack foods often overlook a problem in the packaging design, that is, the packaging is too heavy, many products because of weight or not easy to carry, or the packaging is easily deformed and lost more market opportunities, these are ignored the result of behavioral habits.

(5) The product packaging design and the trend of the times do not fit in today's product packaging design, although it is corporate behavior, there are practical trends in the phenomenon, such as the quiet popularity of environmental protection packaging can not wait and see. Environmental packaging is the most direct reflection of the company's concern for consumers. Such companies are easily recognized by consumers.

\section{Package Design Combined with Consumer Psychology}

\subsection{Specialized design}

The specialization of consumption is the product of social development and economic development. First of all, from the perspective of social stratification, specialized consumption can be seen as a kind of segmentation between different people and different subculture groups. Compared with other consumption, it is not only a kind of conspicuous consumption that invests money, but also a difference between social class and lifestyle. Moreover, with the continuous improvement of people's living standards, the increase in disposable income, and the specialization of consumer knowledge, consumers have turned from blind consumption to rational consumption and professional consumption. Specialized consumption has become an important way for people to promote themselves and gain personal satisfaction. Secondly, through the Internet or other channels, people can form informal groups on consumption. These informal groups are also reference groups that have important influence on participants in the concept of consumption. The breakthrough in science and technology plays an important role in modern packaging. New processes and new technologies provide more and more professional packaging conditions for modern goods. Modern new superb printing and plate making technologies provide more guarantees for achieving perfect results in all aspects of packaging. Art and science and technology are combined more closely, interacting with each other and restricting each other. [4] The use of computer design tools makes it possible for designers to pursue more refined and perfect packaging designs. In the packaging design industry in developed countries and regions, large-scale design units and small-scale design groups complement each other, and the specialization of product companies has to cooperate. Such a mechanism will help high-quality designers play an exemplary role, facilitate information communication and technology exchanges, develop characteristic services, promote design innovation, and meet more specialized packaging design needs in the market. 


\subsection{Diversified design}

The diversification of consumption includes the diversification of consumer subjects and the diversification of objects. Consumers as consumers, there are differences in gender, age, personality, occupation, economy, culture, identity, and other aspects, which determines that consumers have different values in the purchase of goods, and some of the purchase of goods is in order to pursue the use of value, some want to show their status and prestige through the purchase of goods, and others are to pursue the appreciation value of goods. Because of the rich and colorful consumer goods in today's society, people's consumption presents a diversified trend. The diversification of consumption requires that modern packaging design forms also be diversified, so as to meet the needs of consumers of different identities in different ages and industries. In the packaging design style, there are advocates of minimalism, the pursuit of a simple and elegant packaging; there is a claim that packaging design into a strong local flavor of ruralism, with some natural, natural materials and colors, show packaging design is very simple and yet modern style; there are all available scientific and technological means in packaging design to fully demonstrate the beauty of modern science and technology; there is an emphasis on packaging, the environment, the harmony of society and people, everything from people physiological and psychological perspectives to design, to maximize the creation of human comfort and healthy human design; have stressed the design of decorative, while boldly using bright colors and eye-catching words, advocates from the traditional culture to absorb material for creation pursue a kind of humorous postmodernism, etc. [1]. At the same time, modern science and technology also provide the material basis and realization conditions for the diversification of packaging design forms. [4] The new process flow, automation, and computer integrated application provide packaging with more and updated design concepts. The packaging of merchandise on the market is designed using modern science and technology to create a wide variety of levels and dazzling. We are surrounded by a colorful world of packaging. Obviously, we have entered an era of diversified design concepts.

\subsection{Green design}

Green Packaging Design In the 21st century, the rapid development of science and technology, industrial mass production, brought great material satisfaction to people, but at the same time it also brought disaster and shadow to humanity. Today, more and more consumers are advocating energy-saving, environmentally friendly green consumption. Green consumption, also known as sustainable consumption, refers to a new type of consumer behavior and process characterized by moderate control of consumption, avoidance or reduction of environmental damage, advocacy of nature and protection of ecology. It is in line with "3E" and "3R", Economic, Ecological, Equality, Equities, Reducing unnecessary consumption, Reuse and Regeneration. Use (Recycle). Not only includes the purchase and use of green products, but also includes the recycling of materials, the effective use of energy, the protection of living environment, the protection of species and other processes that consume substances and energy [3]. The concept of green packaging design began in the mid-1970s. With the increase of awareness of the environment and ecology protection of the whole society, the design institution has taken ecological protection into the new round of design thinking process from the consideration of the immediate interests and sustainable development of human beings. Green packaging design from the perspective of environmental protection, designed to create a pollution-free, beneficial to human health, is conducive to human survival and reproduction of the ecological environment. For example, the standards of department store packaging formulated by the Japan Department Stores Association Committee: packaging raw materials or devices must not endanger human health, and should minimize the use of packaging materials that are difficult to degrade after being discarded; try to reduce the volume of the packaging. The free volume inside the container should not exceed the product size. Two percent of the volume. The committee also insisted on adopting the simplest packaging method and even required zero packaging efforts. On the other hand, in China's packaging design market, the problem of excessive packaging of goods has been spoken for many years. Not only has it not changed, but it has become increasingly fierce. Green packaging design is not only a technical 
consideration, but also a change of concept. It requires designers to give up the over-emphasis on the product innovation in the product design, and focus on the real sense of the design level. With more concise and lasting styling, the product will be energy-saving and environmentally friendly, and it will convey the green and humanistic spirit [5].

\subsection{Personalized design}

In recent years, with the rapid development of our country's economy, people's living standards and consumption levels have been continuously improved, and values have become more and more personalized, which in turn requires the cultural color and emotional color of goods, and requires that goods reflect the individual's unique qualities. At the same time, more and more goods are available, and supply exceeds demand. Consumers can pick and choose among many similar products. Consumers have become less and less satisfied with stereotyped products. Consumption has become an occasion and opportunity for personal self-expression. Through consumption, you not only show your economic strength, but also your own occupation type, social status, personality traits, personal preferences, and aesthetic perspectives. It can also be said that the current consumption is more concerned with the quality of life and can express itself more subjectively. This trend is reflected in the purchase of goods. Specifically, some people pursue a certain brand, because this brand has become a reflection of his life's taste and personality traits; some people are pursuing trendy and fashionable goods, and they hope that they will always walk in the era. In the front, attention was paid to the people around us; others like to purchase special products that have strong self-esteem. The individualization of consumption is a product of social development and economic development. This phenomenon will also continue to change with the further development of human society. Whether or not a product's packaging design can be enjoyed by the general public will depend to a large extent on whether it has a unique personality that is different from other similar products. At the same time, the enormous wealth of commodities also provides a variety of possibilities for the consumer's personalized consumption. The personalization of consumption requires that packaging design has unique ideas. For example, the Coca-Cola bottle design not only enables consumers to generate brand recognition, but also becomes part of the brand equity, resulting in a huge marketing effect. A bottle turned into a trademark. Coca-Cola believes that in places where people are only in the world, some people know that this is Coca-Cola. The reason is the unique and personalized design of Coca-Cola bottles. Coca-Cola's bottle shape is also known as "the world's most famous bottle." The creative level of packaging design is synchronized with the research level of consumer psychology. The deeper the research on consumer psychology, the higher the level of creativity. Successful packaging design work always embodies the designer's creativity full of personality. It not only reflects the fashion of the times, but also reflects the designer's deep understanding of individual consumer's consciousness, emotions and needs. It can be said that the more people's living standards improve, the more people will be able to produce personalized creative and personalized demands. The two may be formed at the same time [5].

\subsection{Fashionable packaging design}

The stylish packaging design is a dynamic beauty that is constantly changing: Apple's iPod series products seem to have become synonymous with hard disk MP3, and the second-generation iPod has made some adjustments to the packaging design. Lighter, plus five-color shell silver, gold, green, pink and pink blue optional, quality only 104g, more portable, more stylish. Coca-Cola Brazil recently launched a limited-edition Coca-Cola light bottle, which is only available for sale during the "Fashion Week" in Sao Paulo, Brazil. People can only buy this packaged Coca-Cola at the location of Fashion Week. This light bottled capacity of $237 \mathrm{~mL}$, bottle decoration is full of fashion style. This bottle then won the ABRE Trophy of the Brazilian Packaging Design Award and the well-known World Star Award for packaging. Nais's transparent toothpaste packaging uses advanced printing technology and stylish packaging design to demonstrate the market positioning of the product. The fashionable packaging design is always young and always full of life. It represents a new era of cultural information, a manifestation of new life in life, so that the beauty of packaging 
culture has a sense of the times, popularity and popularity. Fashion design that meets the needs of the times can often guide mass consumption, and the demand for mass consumption can often inspire fashion design. The good interaction between the two is actually a good hope for the prospect of fashion design.

\section{Conclusion}

Packaging is an important part of shaping the quality of goods and corporate image, and it is also an economical advertising carrier and competitive tool in corporate marketing. In the competition of similar grades, the competition of goods can be said to be largely a competition between packaging and design. Whether a commodity can enter the market, occupy the market, hold onto the market, in addition to having a good product intrinsic quality, a large one. The role of the aspect depends on the product packaging. With the rapid development of modern science and technology, human beings gradually deepen their understanding of their own values and the surrounding ecological environment. Nowadays, people's consumer psychology is undergoing a qualitative change. This trend requires modern designers must fully recognize the modern consumer psychology and packaging. Design the interdependence relationship between the two, pay attention to the two-way interaction between the two, and strive to create more creativity in the interaction between the two, flying passion, so that modern packaging design can truly meet the needs of people's consumption in the 21st century.

\section{References}

[1] Ma Li. Influence of modern consumer psychology on packaging design[J]. Technology \& Market, 2012, 19(11):98-98.

[2] Yao Jianping. Influence of modern consumer psychology on packaging design[J]. Package Engineering, 2000, 21(1):6-7.

[3] Cao Xinxuan. Research on Product Package Design and Consumer Psychology[J]. Market Modernization, 2008(02): 227.

[4] Jiang Weixi. Application of analytic theme packaging in marketing[D]. Chongqing University, 2010.

[5] Wang Yi, Wang Anxia. Analysis of Individual Consumption Psychology and Its Implications for Packaging Design[J]. Art \& Design (Theory), 2008(12):46-48. 